CERN-EP/2001-014

9 February 2001

\title{
Signal Propagation, Termination, Crosstalk and Losses in Resistive Plate Chambers
}

\author{
Werner Riegler ${ }^{1)}$, Daniel Burgarth ${ }^{2)}$
}

\begin{abstract}
We discuss the signal propagation, strip termination and crosstalk in Resistive Plate Chambers (RPCs) by analyzing the explicit time domain solution of a two dimensional multiconductor transmission line. It is shown that all the effects can be calculated by elementary matrix manipulations.
\end{abstract}

Submitted to Nuclear Instruments and Methods

1) EP Division, CERN, CH-1211 Geneva 23, Switzerland

2) CERN summer student, now at Albert-Ludwigs-Universität 79085 Freiburg, Germany 
In many large size detectors the readout electrodes (strips) are very long i.e. the signal propagation time is large compared to the signal width. In that case the readout electrodes have to be treated as a multi-conductor transmission line. The induced signal acts as current source at some point along the electrode. In order to avoid multiple reflections, the strips have to be terminated properly on at least one end. The strip-end that is connected to the signal amplifiers has to be designed such that the crosstalk is minimized.

In this note we will discuss a RPC with a geometry similar to the design used for $\mathrm{LHCb}$ [1] and ATLAS [2] to illustrate a very powerful formalism for analyzing signal propagation, transmission line termination and crosstalk. The results are of course applicable to any detector geometry that satisfies the requirements for a 2-dimensional transmission line. We will first introduce the general theory and then apply the formalism to some realistic geometries. A more elaborate discussion of RPCs is given in [3].

\section{General Solution}

The theory of multi conductor transmission lines is well developed [4]. We assume here that the width of the readout strips is small compared to their length and that the line is uniform meaning that the geometry is independent of $z$ (Fig. 1). In that case the detector is a 2-dimensional $N$-conductor transmission line and it is completely defined by the $N \times N$ matrices $\hat{\mathbf{C}}, \hat{\mathbf{L}}, \hat{\mathbf{R}}$ and $\hat{\mathbf{G}}$, the 'per unit length' capacitance, inductance, resistance, and transconductance matrix. For the examples given later they were calculated with Maxwell [5], a finite element field simulator program. In case these matrices are independent of frequency (which will be justified in the last chapter), the equations describing the most general 2-dimensional $N$ conductor transmission line in the TEM [4] approximation are

$$
\begin{gathered}
\frac{\partial}{\partial z} \mathbf{V}(z, t)=-\hat{\mathbf{R}} \mathbf{I}(z, t)-\hat{\mathbf{L}} \frac{\partial}{\partial t} \mathbf{I}(z, t) \\
\frac{\partial}{\partial z} \mathbf{I}(z, t)=-\hat{\mathbf{G}} \mathbf{V}(z, t)-\hat{\mathbf{C}} \frac{\partial}{\partial t} \mathbf{V}(z, t)
\end{gathered}
$$

where

$$
\mathbf{I}(z, t)=\left(\begin{array}{c}
I_{1}(z, t) \\
\cdot \\
I_{N}(z, t)
\end{array}\right) \quad \mathbf{V}(z, t)=\left(\begin{array}{c}
V_{1}(z, t) \\
\cdot \\
V_{N}(z, t)
\end{array}\right)
$$

are the currents and voltages of the $N$ individual conductors at time $t$ and position $z$ along the transmission line. If losses can be neglected (which will be justified in the last chapter) the

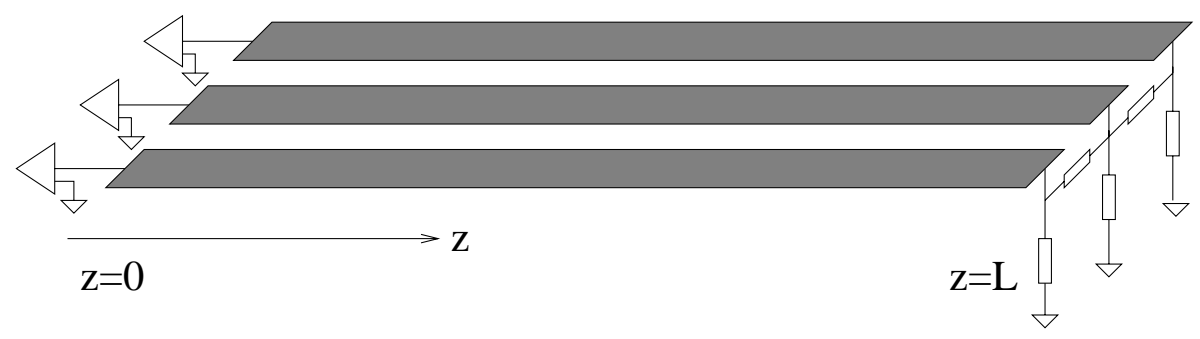

Figure 1: 2-dimensional multi-conductor transmission line. The crossection has to be independent of $z$. The shape of the individual conductors can however be different. 
matrices $\hat{\mathbf{R}}$ and $\hat{\mathbf{G}}$ are zero and the above equations simplify to

$$
\frac{d^{2}}{d z^{2}} \mathbf{I}(z, t)=\hat{\mathbf{C}} \hat{\mathbf{L}} \frac{d^{2}}{d t^{2}} \mathbf{I}(z, t) \quad \frac{d^{2}}{d z^{2}} \mathbf{V}(z, t)=\hat{\mathbf{L}} \hat{\mathbf{C}} \frac{d^{2}}{d t^{2}} \mathbf{V}(z, t)
$$

The general solution of these equations is

$$
\begin{aligned}
& \mathbf{I}(z, t)=\hat{\mathbf{T}}\left(\left(\begin{array}{c}
I_{1}^{+}\left(t-\frac{z}{v_{1}}\right) \\
\cdot \\
I_{N}^{+}\left(t-\frac{z}{v_{N}}\right)
\end{array}\right)-\left(\begin{array}{c}
I_{1}^{-}\left(t+\frac{z}{v_{1}}\right) \\
\cdot \\
I_{N}^{-}\left(t+\frac{z}{v_{N}}\right)
\end{array}\right)\right) \\
& \mathbf{V}(z, t)=\hat{\mathbf{Z}}_{C} \hat{\mathbf{T}}\left(\left(\begin{array}{c}
I_{1}^{+}\left(t-\frac{z}{v_{1}}\right) \\
\cdot \\
I_{N}^{+}\left(t-\frac{z}{v_{N}}\right)
\end{array}\right)+\left(\begin{array}{c}
I_{1}^{-}\left(t+\frac{z}{v_{1}}\right) \\
\cdot \cdot z \\
I_{N}^{-}\left(t+\frac{z}{v_{N}}\right)
\end{array}\right)\right)
\end{aligned}
$$

where the $I_{m}^{+}(x)$ and $I_{m}^{-}(x)$ are $2 N$ arbitrary functions and

$$
\hat{\mathbf{T}}^{-1}(\hat{\mathbf{C}} \hat{\mathbf{L}}) \hat{\mathbf{T}}=\hat{\mathbf{v}}^{-2} \quad \hat{\mathbf{Z}}_{C}=\sqrt{\hat{\mathbf{L}} / \hat{\mathbf{C}}}=\hat{\mathbf{L}} \hat{\mathbf{T}} \hat{\mathbf{v}} \hat{\mathbf{T}}^{-1}
$$

with

$$
\hat{\mathbf{v}}^{-2}=\left(\begin{array}{ccc}
\frac{1}{v_{1}^{2}} & \cdot & 0 \\
\cdot & \cdot & \cdot \\
0 & \cdot & \frac{1}{v_{N}^{2}}
\end{array}\right) \quad \hat{\mathbf{v}}=\left(\begin{array}{ccc}
v_{1} & \cdot & 0 \\
\cdot & \cdot & \cdot \\
0 & \cdot & v_{N}
\end{array}\right)
$$

The matrix $\hat{\mathbf{T}}$ contains the normalized eigenvectors of the matrix $\hat{\mathbf{C}} \hat{\mathbf{L}}$ and $1 / v_{i}^{2}$ are the corresponding eigenvalues. The matrix $\hat{\mathbf{Z}}_{C}$ is called the characteristic impedance matrix. The individual functions represent pulses that are running along the strips in positive and negative direction without changing their shape. Note that in general the signal propagation happens with $N$ different velocities and also note that the solution is completely general.

The explicit form of these functions is determined by the line excitation mechanism and boundary conditions at the strips ends $z=0$ and $z=L$. A detector signal acts as an ideal current source $I^{0}(t)$ at a position $z=z_{0}$ somewhere along a conductor $n$, which defines the $2 N$ functions. Hence we have the general solution

$$
\begin{aligned}
& \mathbf{I}(z, t)=\frac{1}{2} \hat{\mathbf{T}}\left(\left(\begin{array}{c}
t_{1 n}^{-1} I^{0}\left(t-\frac{z-z_{0}}{v_{1}}\right) \\
\cdot \\
t_{N n}^{-1} I^{0}\left(t-\frac{z-z_{0}}{v_{N}}\right)
\end{array}\right)-\left(\begin{array}{c}
t_{1 n}^{-1} I^{0}\left(t+\frac{z-z_{0}}{v_{1}}\right) \\
\cdot \\
t_{N n}^{-1} I^{0}\left(t+\frac{z-z_{0}}{v_{N}}\right)
\end{array}\right)\right) \\
& \mathbf{V}(z, t)=\frac{1}{2} \hat{\mathbf{Z}}_{C} \hat{\mathbf{T}}\left(\left(\begin{array}{c}
t_{1 n}^{-1} I^{0}\left(t-\frac{z-z_{0}}{v_{1}}\right) \\
\cdot \\
t_{N n}^{-1} I^{0}\left(t-\frac{z-z_{0}}{v_{N}}\right)
\end{array}\right)+\left(\begin{array}{c}
t_{1 n}^{-1} I^{0}\left(t+\frac{z-z_{0}}{v_{1}}\right) \\
\cdot \\
t_{N n}^{-1} I^{0}\left(t+\frac{z-z_{0}}{v_{N}}\right)
\end{array}\right)\right)
\end{aligned}
$$

which we can write as

$$
\begin{gathered}
\mathbf{I}(z, t)=\mathbf{I}^{+}(z, t)-\mathbf{I}^{-}(z, t) \\
\mathbf{V}(z, t)=\hat{\mathbf{Z}}_{C}\left[\mathbf{I}^{+}(z, t)+\mathbf{I}^{-}(z, t)\right]=\mathbf{V}^{+}(z, t)+\mathbf{V}^{-}(z, t)
\end{gathered}
$$

The $t_{n m}^{-1}$ are the elements of the matrix $\hat{\mathbf{T}}^{-1}$. It is easy to see that at $z=z_{0}$ it holds that $\mathbf{I}^{+}\left(z_{0}, t\right)+\mathbf{I}^{-}\left(z_{0}, t\right)=\left(0, ., I^{0}(t), ., 0\right)^{T}$, so it satisfies the required boundary condition. This solution shows that there are pulses running symmetrically in the positive and negative direction 
from the point $z_{0}$. The pulse running along one conductor is a superposition of $N$ times the same pulse-shape $I^{0}(t)$ running with $N$ different velocities $v_{i}$. Therefore we find signal dispersion even for a lossless transmission line which is called modal dispersion.

The pulses will travel until they hit the strip ends where they are reflected according to the connected networks. We assume now an arbitrary interconnection of strips at $z=0$ and $z=L$ with purely resistive loads. For $z=L$ we define $R_{i j} i \neq j$ the resistors between strip $i$ and $j$ and $R_{i i}$ the resistors between strip $i$ and ground. The boundary condition is then given by

$$
\begin{array}{ll}
\mathbf{V}(L, t)=\hat{\mathbf{Z}}_{T} \mathbf{I}(L, t) & \hat{\mathbf{Z}}_{T}=\hat{\mathbf{Y}}_{T}^{-1} \\
Y_{i j}^{T}=-\frac{1}{R_{i j}} \quad i \neq j & Y_{i i}^{T}=\sum_{j=1}^{N} \frac{1}{R_{i j}}
\end{array}
$$

where we define $\hat{\mathbf{Z}}_{T}$ as the load impedance matrix. The other strip end at $z=0$ will of course be characterized by a different load impedance matrix which we call $\hat{\mathbf{Z}}_{P}$ since we assume it is the readout (preamp) side. The effect of the boundary is that the voltage pulses are reflected according to

$$
\mathbf{V}_{r e f l}^{-}=\hat{\Gamma}_{T} \mathbf{V}^{+} \quad \text { and } \quad \mathbf{V}_{r e f l}^{+}=\hat{\Gamma}_{P} \mathbf{V}^{-}
$$

where the reflection coefficient matrix $\hat{\Gamma}$ at the line ends is defined as

$$
\hat{\boldsymbol{\Gamma}}_{T}=\left(\hat{\mathbf{Z}}_{T}-\hat{\mathbf{Z}}_{C}\right)\left(\hat{\mathbf{Z}}_{T}+\hat{\mathbf{Z}}_{C}\right)^{-1} \quad \hat{\boldsymbol{\Gamma}}_{P}=\left(\hat{\mathbf{Z}}_{P}-\hat{\mathbf{Z}}_{C}\right)\left(\hat{\mathbf{Z}}_{P}+\hat{\mathbf{Z}}_{C}\right)^{-1}
$$

and the actual voltages at the strip ends are given by

$$
\begin{aligned}
& \mathbf{V}(L, t)=\mathbf{V}^{+}+\mathbf{V}_{r e f l}^{-}=\left(\hat{\mathbf{1}}+\hat{\boldsymbol{\Gamma}}_{T}\right) \mathbf{V}^{+} \\
& \mathbf{V}(0, t)=\mathbf{V}^{-}+\mathbf{V}_{r e f l}^{+}=\left(\hat{\mathbf{1}}+\hat{\boldsymbol{\Gamma}}_{P}\right) \mathbf{V}^{-}
\end{aligned}
$$

This is our final solution. Given the current pulse $I^{0}(t)$ at position $z=z_{0}$ on conductor $n$ we know the two pulses $\mathbf{V}^{+}$and $\mathbf{V}^{-}$running symmetrically in both directions from $z=z_{0}$ towards the two line ends from Eq. 4. The networks at the line ends define the matrices $\hat{\boldsymbol{\Gamma}}_{T}$ and $\hat{\boldsymbol{\Gamma}}_{P}$ which give the reflected and measured pulses. If the transmission line is not terminated we of course have to add up the multiple reflections.

\section{Termination}

If we want to eliminate reflections at the line end $z=L$ the reflection coefficient matrix $\Gamma_{T}$ has to be zero, i.e. the load impedance matrix $\hat{\mathbf{Z}}_{T}$ has to be equal to the characteristic impedance matrix $\hat{\mathbf{Z}}_{C}$. The termination resistors $R_{i j}^{T}$ are calculated by inverting the Eq. 5 evaluating to

$$
\hat{\mathbf{Y}}_{C}=\hat{\mathbf{Z}}_{C}^{-1} \quad R_{i j}^{T}=-\frac{1}{Y_{i j}^{C}} \quad i \neq j \quad \frac{1}{R_{i i}^{T}}=\sum_{j=0}^{N} Y_{i j}^{C}
$$

We see that in order to eliminate reflections we theoretically have to interconnect all the conductors i.e. we need $\frac{1}{2} N(N+1)$ termination resistors. Examples will be discussed later.

\section{$4 \quad$ Measured Signal}

Now we assume that one end $(z=L)$ of the transmission line is perfectly terminated. The other end $(z=0)$ is read by amplifiers and is loaded by $\hat{\mathbf{Z}}_{P}$. If the current $I^{0}(t)$ is induced 
on strip $n$, the voltage and current measured by the amplifiers is calculated from Eq. 4 and 7 which gives

$$
\mathbf{V}_{\text {meas }}(t)=\mathbf{V}(0, t)=\hat{\mathbf{Z}}_{P} \hat{\mathbf{Z}}_{C}\left(\hat{\mathbf{Z}}_{P}+\hat{\mathbf{Z}}_{C}\right)^{-1} \hat{\mathbf{T}}\left(\begin{array}{c}
t_{1 n}^{-1} I^{0}\left(t-\frac{z_{0}}{v_{1}}\right) \\
t_{N n}^{-1} I^{0}\left(t-\frac{z_{0}}{v_{N}}\right)
\end{array}\right)
$$

and

$$
\mathbf{I}_{\text {meas }}(t)=\frac{1}{R_{\text {in }}} \mathbf{V}_{\text {meas }}(t) \neq \mathbf{I}(0, t)
$$

$R_{\text {in }}$ is the amplifier input resistance. $\mathbf{I}(0, t)$ is the current at the line end which is different from the current flowing through the amplifier in case the strips are interconnected. The relative amplitudes of the voltages give the crosstalk which we discuss next.

\section{$5 \quad$ Crosstalk}

The above solution allows us to write down the explicit formula for the crosstalk from the signal strip $n$ to all other strips.

\subsection{Homogeneous and Inhomogeneous Transmission Lines}

In case the volume, where the electro-magnetic waves propagate, has uniform dielectric properties, all the propagation velocities are the same and we call the geometry a homogeneous transmission line. It then holds that

$$
\hat{\mathbf{L}} \hat{\mathbf{C}}=\frac{1}{v^{2}} \hat{\mathbf{1}} \quad \hat{\mathbf{Z}}_{C}=v \hat{\mathbf{L}}
$$

An example is the geometry shown in Fig. 3. The RPC geometry that we want to study (Fig. 9) is however an inhomogeneous transmission line and we will therefore find $N$ different propagation velocities causing signal dispersion even if the transmission line is lossless.

\subsection{Transmission Line with Small Dispersion}

If all the propagation velocities are the same or if the transmission line is short, such that the dispersion is very small, the above solution evaluates to

$$
\mathbf{V}_{\text {meas }}(t)=\hat{\mathbf{Z}}_{P} \hat{\mathbf{Z}}_{C}\left(\hat{\mathbf{Z}}_{P}+\hat{\mathbf{Z}}_{C}\right)^{-1}\left(0, ., 0, I^{0}\left(t-\frac{z_{0}}{v}\right), 0, ., 0\right)^{T}
$$

Defining the Matrix $\hat{\mathbf{M}}=\hat{\mathbf{Z}}_{P} \hat{\mathbf{Z}}_{C}\left(\hat{\mathbf{Z}}_{P}+\hat{\mathbf{Z}}_{C}\right)^{-1}$ the crosstalk from conductor $n$ to conductor $m$ is given by $V_{m} / V_{n}=M_{m n} / M_{n n}$. If we adjust the preamp input resistance and interconnecting resistors such that $\hat{\mathbf{Z}}_{P}=\hat{\mathbf{Z}}_{C}$ i.e. if we ideally terminate the preamp side, the solution is given by

$$
\mathbf{V}_{\text {meas }}(t)=\frac{1}{2} \hat{\mathbf{Z}}_{C}\left(0, ., 0, I^{0}\left(t-\frac{z_{0}}{v}\right), 0, ., 0\right)^{T}
$$

The crosstalk from signal strip $n$ to strip $m$ is then given by $Z_{n m}^{C} / Z_{n n}^{C}$. We see that in order to have small crosstalk, the off-diagonal elements in the characteristic impedance matrix $\hat{\mathbf{Z}}_{C}$ should be small compared to the diagonal elements. Terminating the amplifier side is however not the optimum scenario in terms of collected charge and crosstalk which will be shown next.

If we do not interconnect the strips on the amplifier side but just connect each strip to the preamp, it holds that $\hat{\mathbf{Z}}_{P}=\operatorname{Diag}\left(R_{i n}, R_{i n}, \ldots, R_{i n}\right)$ where $R_{\text {in }}$ is the preamp input resistance. In case the amplifier input resistance is $R_{\text {in }}=0$ we have $\hat{\mathbf{Z}}_{P}=0, \mathbf{V}_{\text {meas }}(t)=0$ and

$$
I_{\text {meas }}(t)=\left(0,0, \ldots, I^{0}\left(t-\frac{z_{0}}{v}\right), \ldots, 0\right)^{T}
$$


i.e. we measure exactly the pulse induced on line $n$ and zero on all the other lines. The whole scenario looks the following: If a current pulse is induced at point $z=z_{0}$, half of it runs to the left and half of it to the right. The pulse running to the right is absorbed in the termination network $(z=L)$, the pulse running to the left is totally negatively reflected $(z=0)$ and the amplifiers measure the difference i.e. the entire current signal. The reflection again runs to the right where it is absorbed. This way we measure the maximum signal with minimum crosstalk. It can be shown that interconnecting the strips on the preamp side i.e. introducing off-diagonal elements in the load impedance matrix $\hat{\mathbf{Z}}_{P}$, will always increase the crosstalk.

Therefore we conclude for a terminated transmission line with small dispersion that the measured signals on all strips have the same shape as the original induced signal, the crosstalk is independent of the position of the induced signal along the strip, the signal will be maximal and the crosstalk minimal if we do not interconnect the strips and the amplifier input resistance $R_{\text {in }}$ is zero (or lowest possible).

\subsection{Transmission Line with Significant Dispersion}

For a long, inhomogeneous transmission line the individual pulses will disperse as they run along the strips and the pulse-shapes will change. The crosstalk will therefore in general increase as a function of distance from the amplifier and will also depend on the shape of the induced signal. If we integrate the current flowing through the amplifier, the dispersion effect vanishes and we find

$$
\mathbf{Q}=\int \mathbf{I}_{\text {meas }}(t) d t=\frac{1}{R_{\text {in }}} \hat{\mathbf{Z}}_{P} \hat{\mathbf{Z}}_{C}\left(\hat{\mathbf{Z}}_{P}+\hat{\mathbf{Z}}_{C}\right)^{-1}(0, ., 0, q, 0, ., 0)^{T}
$$

where $q=\int I^{0}(t) d t$ is the total charge induced on strip $n$, so the charge fraction measured on the neighbouring strips ('crosstalk charge') is given as before by $Q_{m} / Q_{n}=M_{m n} / M_{n n}$. This will be the observed crosstalk for 'slow' readout electronics i.e. amplifiers with an integration time that is much larger than the signal dispersion time. Fast amplifiers will however sense the signal dispersion and will therefore show more crosstalk. In case $R_{i n}=0$ the crosstalk charge is zero which means that all the crosstalk signals are perfectly bipolar.

We conclude on the transmission line with significant dispersion that the signal shapes change as a function of distance from the preamp and are only equal to the original induced signal if the current is induced close to the preamp. Therefore the amplitude and shape of the crosstalk signal also changes as a function of distance from the amplifier. In general the crosstalk will increase as a function of distance from the amplifier. The crosstalk is lowest if the strips are not interconnected and the amplifier input resistance is as low as possible. The crosstalk is smaller for slow electronics. 


\section{$6 \quad$ Examples}

In this section we apply the formalism to an actual RPC geometry. To study all aspects we discuss a single strip RPC, a homogeneous double strip transmission line, a double strip RPC and finally a RPC with many strips and guard strip.

\subsection{Single Strip RPC}

A single strip RPC is shown in Fig. 2. The parameters calculated with Maxwell are

$$
C=205 \mathrm{pF} / \mathrm{m} \quad L=89.3 \mathrm{nH} / \mathrm{m} \quad \Rightarrow \quad Z_{C}=20.87 \Omega \quad v=2.34 \times 10^{8} \mathrm{~m} / \mathrm{s}
$$

The strip is terminated simply by putting a termination resistor $R_{T}=Z_{C}$ at the strip end $z=L$. The signal measured by an amplifier at $z=0$ with input resistance $R_{i n}$ is given by

$$
I_{\text {meas }}(t)=\frac{Z_{C}}{Z_{C}+R_{\text {in }}} I^{0}\left(t-\frac{z_{0}}{v}\right)
$$

so it has the same shape as the original induced signal, independent of $z_{0}$. In case the preamp input impedance is $R_{i n}=0$ the measured signal is equal to the induced signal. This was easy.

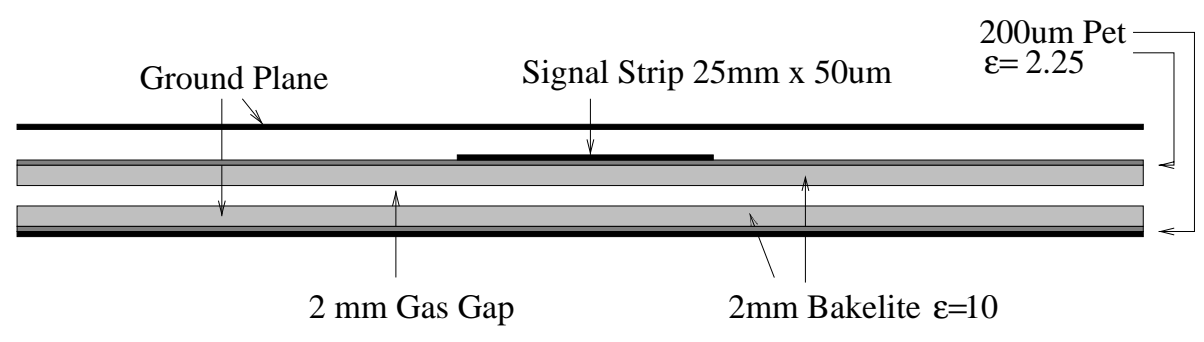

Figure 2: Crossection through a RPC with a single signal strip. The current signal is induced by the avalanche electrons moving in the gas gap. 


\subsection{Homogeneous Double Strip Line}

To see the difference between an homogeneous and inhomogeneous transmission line we study the above RPC geometry with two strips and first omit the Bakelite (Fig. 3). In that case the dielectric properties are equal in the entire area where the waves propagate and the propagation velocity will be equal for all waves. The Maxwell calculations give

$$
\begin{array}{lll}
\hat{\mathbf{C}}=\left(\begin{array}{cc}
126 & -6.4 \\
-6.4 & 126
\end{array}\right) \mathrm{pF} / \mathrm{m} & \hat{\mathbf{L}}=\left(\begin{array}{cc}
88.4 & 4.47 \\
4.47 & 88.4
\end{array}\right) \mathrm{nH} / \mathrm{m} \Rightarrow \\
\hat{\mathbf{Z}}_{C}=\left(\begin{array}{cc}
26.5 & 1.34 \\
1.34 & 26.5
\end{array}\right) \Omega & \hat{\mathbf{R}}_{T}=\left(\begin{array}{cc}
27.8 & 522.7 \\
522.7 & 27.8
\end{array}\right) \Omega & v=3 \times 10^{8} \mathrm{~m} / \mathrm{s}
\end{array}
$$

The capacitance matrix $\hat{\mathbf{C}}$ is defined such that the negative off-diagonal element $-C_{i j}$ is the mutual capacitance between conductor $i$ and $j$, and the sum of the $i^{\text {th }}$ column $\sum_{j} C_{i j}$ is the capacitance of conductor $i$ to ground. Since the matrix $\hat{\mathbf{C}} \hat{\mathbf{L}}$ is already diagonal the matrix $\hat{\mathbf{T}}$ is undefined and any set of two orthonormal vectors will do for it. The matrix $\hat{\mathbf{R}}_{T}$ contains the termination resistors calculated from Eq. 8. Only if we interconnect the strips we avoid reflections (Fig. 4). One side of the strips we finally want to read out with an amplifier of input resistance $R_{i n}$. Since the lines are not interconnected the load impedance matrix is a diagonal matrix with $R_{i n}$ as diagonal elements. The measured current for an induced current pulse $I^{0}(t)$ at $z=z_{0}$ of strip 1 is given by Eq. 9 and evaluates to

$$
\left(\begin{array}{c}
I_{\text {meas }}^{1}(t) \\
I_{\text {meas }}^{2}(t)
\end{array}\right)=\frac{1}{R_{\text {in }}^{2}+2 R_{\text {in }} Z_{11}+Z_{11}^{2}-Z_{12}^{2}}\left(\begin{array}{c}
R_{\text {in }} Z_{11}+Z_{11}^{2}-Z_{12}^{2} \\
R_{\text {in }} Z_{12}
\end{array}\right) I^{0}\left(t-\frac{z_{0}}{c}\right)
$$

so we find a crosstalk of

$$
\frac{I_{\text {meas }}^{2}(t)}{I_{\text {meas }}^{1}(t)}=\frac{R_{\text {in }} Z_{12}}{R_{\text {in }} Z_{11}+Z_{11}^{2}-Z_{12}^{2}}
$$

which is illustrated in Fig. 5. The measured signals on both strips have exactly the same shape as the original induced signal $I^{0}(t)$. The crosstalk is zero if the preamp input resistance is zero. In that case we measure exactly the induced current signal. In order to keep the crosstalk small we want the ratio $Z_{12} / Z_{11}$ to be small, so the off-diagonal elements in the impedance matrix should be small compared to the diagonal ones. In the limit of $R_{i n} \rightarrow \infty$ the crosstalk goes to $Z_{12} / Z_{11}$ and the pulse-height goes to zero.

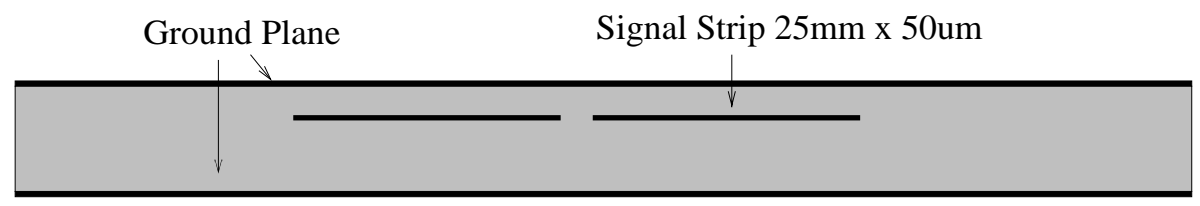

Strip-Strip Distance 2mm

Figure 3: Crossection through a homogeneous transmission line with two strips. 


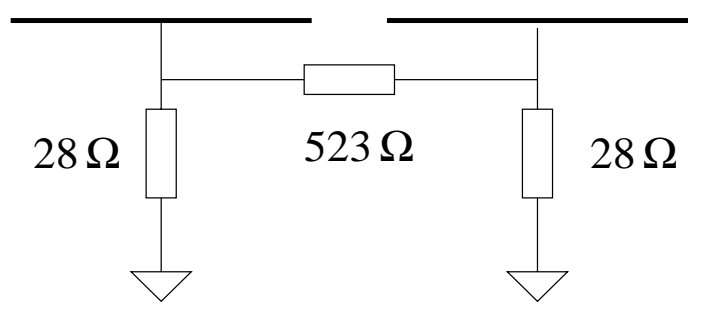

Figure 4: Termination network for a double strip RPC. In order to perfectly terminate a multiconductor transmission line one has to interconnect all the lines.
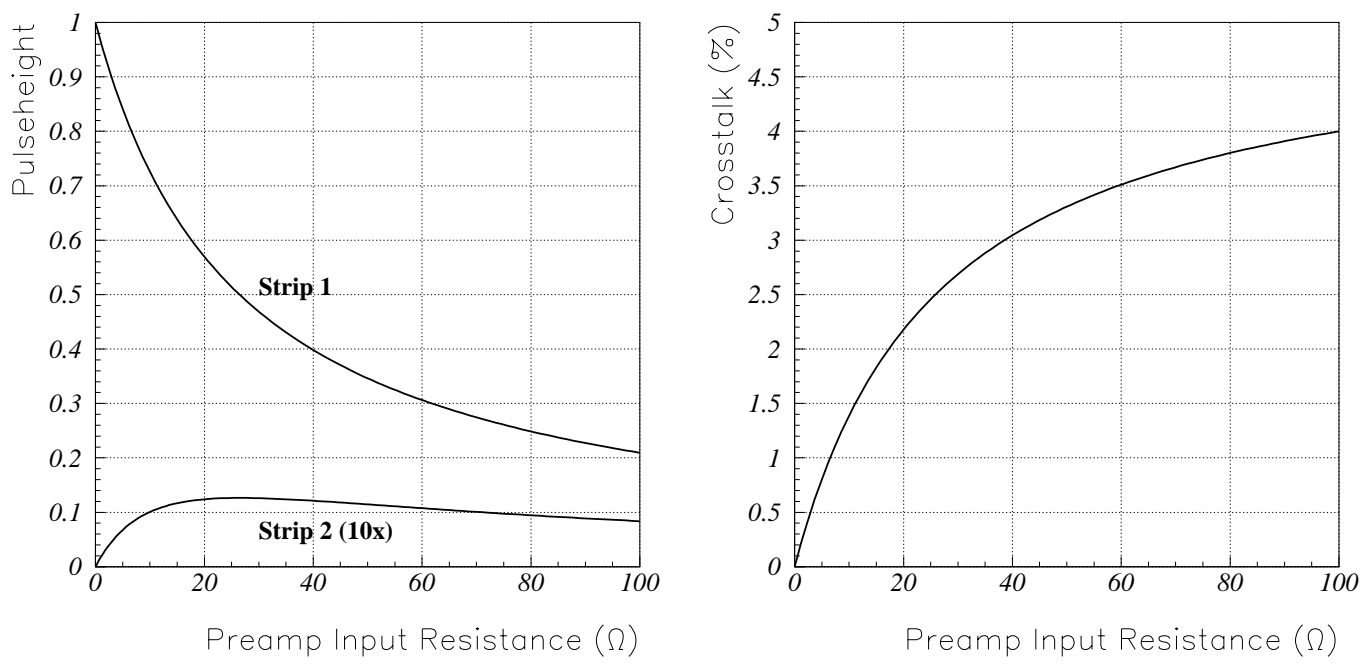

Figure 5: The first figure shows the pulse-height on both strips (the crosstalk pulse is multiplied by 10 for illustration) The current is induced on strip 1 . The second figure shows the crosstalk. We see that the pulse-height decreases and the crosstalk increases for larger preamp input resistance. 


\subsection{Double Strip RPC}

Adding the Bakelite to the geometry discussed above (Fig. 6) we find the characteristic parameters

$$
\begin{array}{ll}
\hat{\mathbf{C}}=\left(\begin{array}{cc}
216 & -30 \\
-30 & 216
\end{array}\right) \mathrm{pF} / \mathrm{cm} & \hat{\mathbf{L}}=\left(\begin{array}{cc}
88.4 & 4.47 \\
4.47 & 88.4
\end{array}\right) \mathrm{nH} / \mathrm{m} \Rightarrow \\
\hat{\mathbf{Z}}_{C}=\left(\begin{array}{cc}
20.4 & 1.93 \\
1.93 & 20.4
\end{array}\right) \Omega & \hat{\mathbf{T}}=\frac{1}{\sqrt{2}}\left(\begin{array}{cc}
1 & 1 \\
-1 & 1
\end{array}\right) \\
\hat{\mathbf{R}}_{T}=\left(\begin{array}{cc}
22.3 & 213.7 \\
213.7 & 22.3
\end{array}\right) \Omega & \hat{\mathbf{v}}=\left(\begin{array}{cc}
2.2 & 0 \\
0 & 2.4
\end{array}\right) \times 10^{8} \mathrm{~m} / \mathrm{s}
\end{array}
$$

The Bakelite has increased the mutual strip-strip capacitance from 6.4 to $30 \mathrm{pF} / \mathrm{m}$ but has left the inductance unchanged (as expected). Therefore the off-diagonal elements in the impedance matrix are larger which will increase the crosstalk. the propagation velocities of the two modes differ by $10 \%$. For a signal $I^{0}(t)$ at $z=z_{0}$ the preamps measure a current of

$$
\begin{gathered}
\left(\begin{array}{c}
I_{\text {meas }}^{1}(t) \\
I_{\text {meas }}^{2}(t)
\end{array}\right)=\frac{1}{2} c_{1}\left(\begin{array}{cc}
R_{i n} Z_{11}+Z_{11}^{2}-Z_{12}^{2} & R_{\text {in }} Z_{12} \\
R_{\text {in }} Z_{12} & R_{\text {in }} Z_{11}+Z_{11}^{2}-Z_{12}^{2}
\end{array}\right)\left(\begin{array}{c}
I^{0}\left(t-\frac{z_{0}}{v_{1}}\right)+I^{0}\left(t-\frac{z_{0}}{v_{2}}\right) \\
-I^{0}\left(t-\frac{z_{0}}{v_{1}}\right)+I^{0}\left(t-\frac{z_{0}}{v_{2}}\right)
\end{array}\right) \\
c_{1}=\frac{1}{R_{\text {in }}^{2}+2 R_{\text {in }} Z_{11}+Z_{11}^{2}-Z_{12}^{2}}
\end{gathered}
$$

We see that the measured signal is a superposition (according to the matrix $\hat{\mathbf{T}}$ ) of two pulses with the same shape running with two different velocities. Due to the dispersion the crosstalk will depend on the pulse-shape $I^{0}(t)$ and the amplifier response. We assume a current pulse-shape of

$$
I^{0}(t)=E_{w} e_{0} v_{e} N_{0}\left(1-\frac{t v_{e}}{d}\right) e^{\alpha v_{e} t} \quad 0<t<d / v_{e}
$$

where $v_{e}$ is the electron drift-velocity, $\alpha$ is the Townsend coefficient, $d$ is the thickness of the gas gap, $N_{0}$ is the number of primary electrons that are uniformly distributed along the track and $E_{w}$ is the weighting field of the electrode. For the following calculations we assume $v_{e}=100 \mu \mathrm{m} / \mathrm{ns}, \alpha=100 \mathrm{~cm}^{-1}$ and $d=2 \mathrm{~mm}$. The dispersion effect is illustrated in Fig. 7. If the strips are short or the signal is induced close to the preamp side we can neglect the different

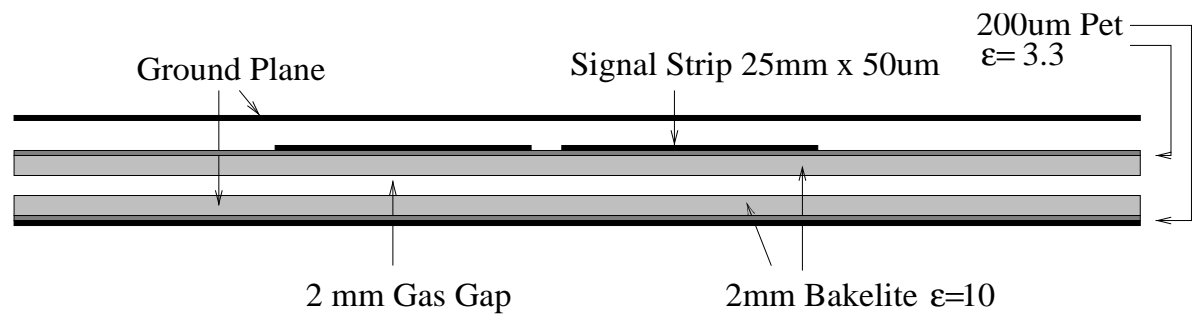

Strip-Strip Distance $2 \mathrm{~mm}$

Figure 6: RPC with two strips. The dielectric properties are not uniform in the area where the waves propagate which leads to different propagation velocities. 
propagation times and the crosstalk is again given by Eq. 11. In that case the crosstalk is independent of the peaking time and the crosstalk signal has the same shape as the actual induced signal.

For long strips the crosstalk will depend on the amplifier response which we assume as

$$
f(t)=\left(\frac{n t}{t_{p}}\right)^{n} e^{-\frac{n t}{t_{p}}}
$$

where $t_{p}$ is the peaking time of the amplifier and $n$ is the number of integrations stages. In the following we will assume $n=3$. The measured signal is given by the convolution of the current signal with the delta response $f(t)$. The result is illustrated in Fig. 8. We find a very strong dependence of the crosstalk on the amplifier peaking time and the distance. 

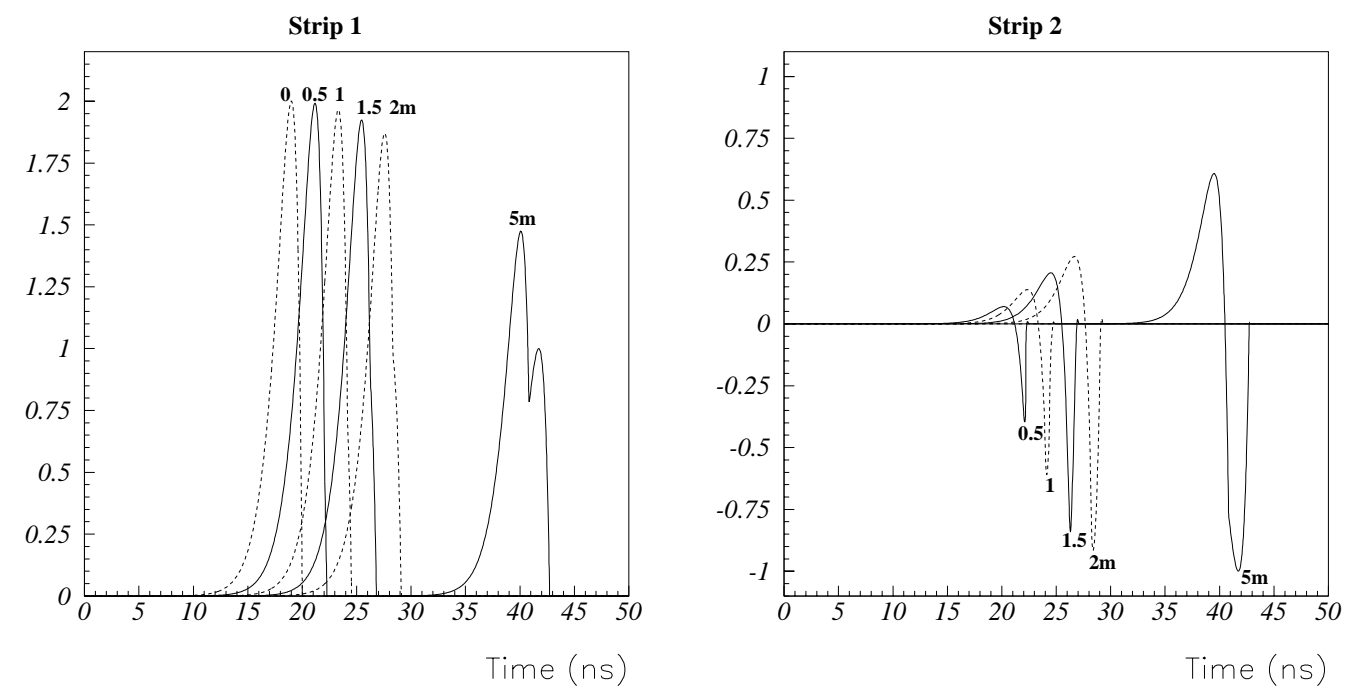

Figure 7: Current pulses running along the two strips of the RPC shown in Fig. 6. The first plot shows the signal strip. One can see that the two 'modes' are dispersing after some distance. The second plot shows the signal travelling on the neighbouring strip. At the position where the signal is induced the crosstalk signal is zero, as the modes are dispersing the crosstalk increases.

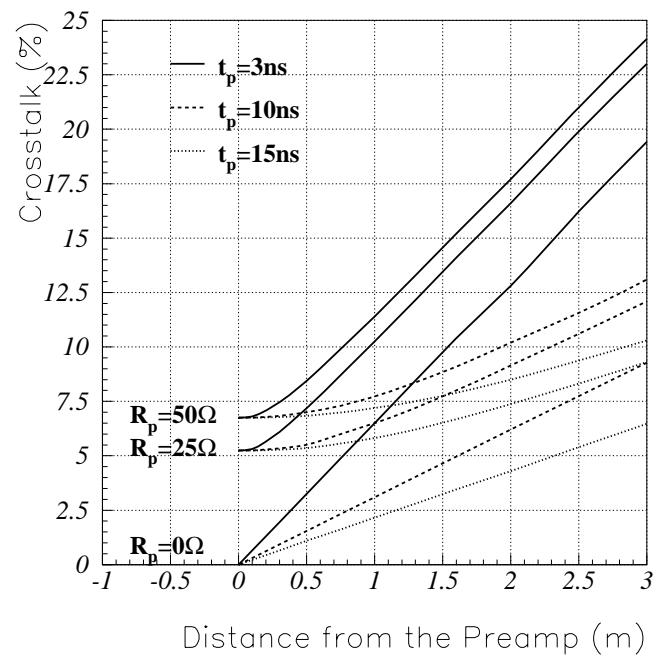

Figure 8: Crosstalk for different preamp input resistances and peaking times as a function of distance from the amplifier side (RPC shown in Fig. 6). For small distances the pulses do not disperse and the crosstalk is given by Eq. 11, independent of the amplifier speed. For fast amplifiers the crosstalk increases strongly with the distance. In the limit of very long peaking times the crosstalk would become independent of the position. 


\subsection{RPC with many Strips and Guard Strip}

Finally we investigate the crosstalk for an RPC with many strips and an additional guard strip in between the signal strips (Fig. 9). Due to the guard strip the cross capacitance between two signal strips reduces from 30 to $21.7 \mathrm{pF} / \mathrm{m}$. The ideal termination network is shown in Fig. 10. All other interconnections are $>25 \mathrm{k} \Omega$ and can be neglected. It is important that the guard strip is not grounded but also included in the termination network on the 'far' side if we want to avoid any reflections. To illustrate the effect Fig. 11 shows the scenario where the signal strips are connected to ground with $25 \Omega$, the strips are not interconnected and the guard strip is grounded on both sides (preamp and termination side). As expected we find reflections. The significance of the reflections and the question about how many termination interconnections in Fig. 10 are therefore necessary has of course to be decided for the actual application.

Connecting the strips to amplifiers with input resistance $R_{i n}$ and grounding the guard strips gives the load impendance matrix $\hat{\mathbf{Z}}_{P}=\operatorname{Diag}\left(\ldots, R_{i n}, 0, R_{i n}, 0, R_{i n} \ldots\right)$. The crosstalk versus distance to the first and second neighbour is shown in Fig. 12. The mutual capacitance to the second neighbour is very small, i.e. the crosstalk to the second neighbour happens mainly through the first neighbour. Fig. 13 shows the crosstalk signal for two different distances of the induced signal from the preamplifier side. As discussed before the shape of the crosstalk signal changes as a function of distance.

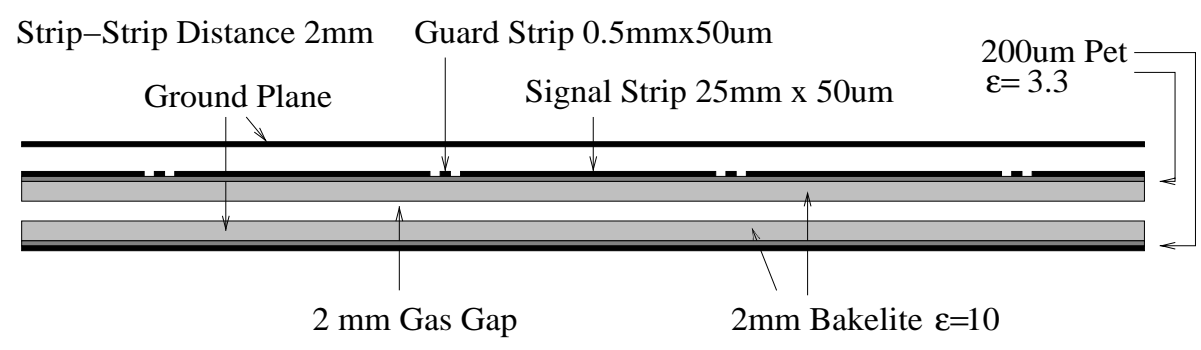

Figure 9: RPC geometry with many strips and a guard strip to reduce the strip-strip coupling. 


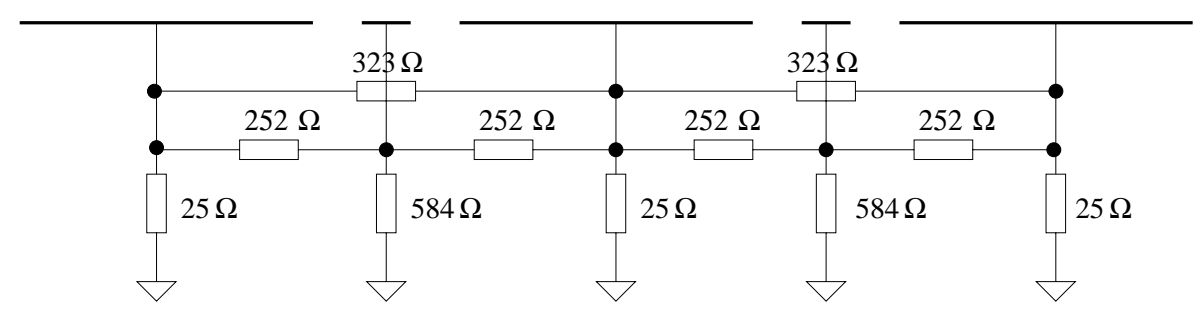

Figure 10: Ideal termination for the RPC with many strips. All other interconnections are $>$ $25 \mathrm{k} \Omega$ and have negligible effect.

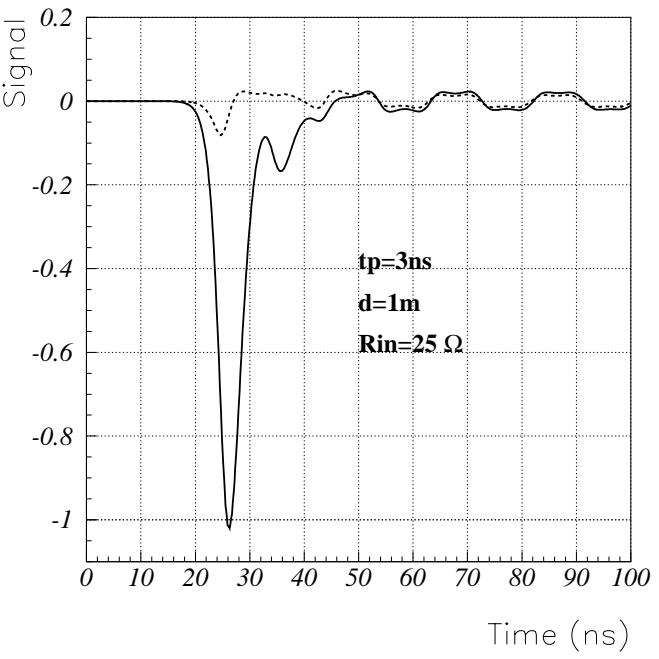

Figure 11: Signal for the scenario where the RPC strips are terminated at $25 \Omega$, the intermediate strips are grounded on both sides and the strips are not interconnected. As expected we find reflections. 


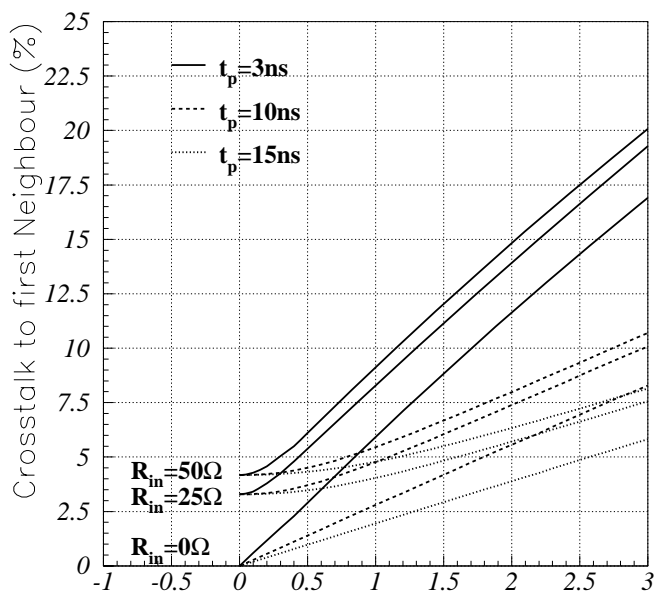

Distance from the Preamp (m)

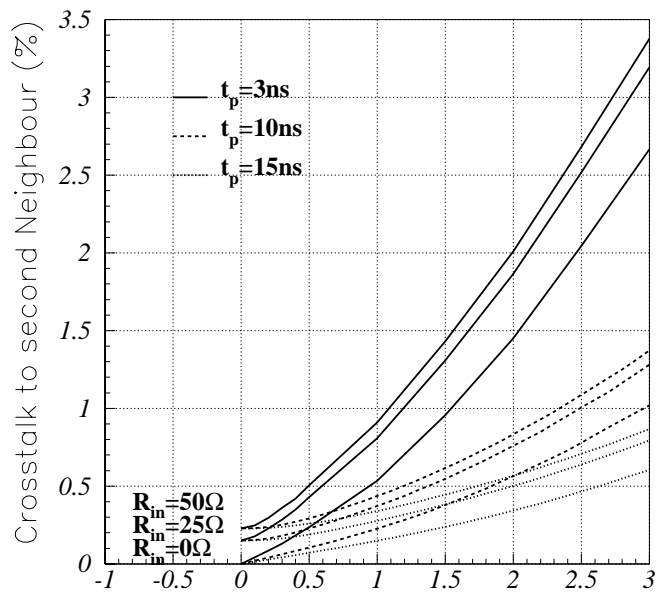

Distance from the Preamp ( $m$ )

Figure 12: Crosstalk to the first and second neighbour for different peaking times and preamp input resistances. The crosstalk to the second neighbour happens mainly through the first neighbour and not through direct coupling which can be seen by the fact that the second plot is approximately the square of the first one.
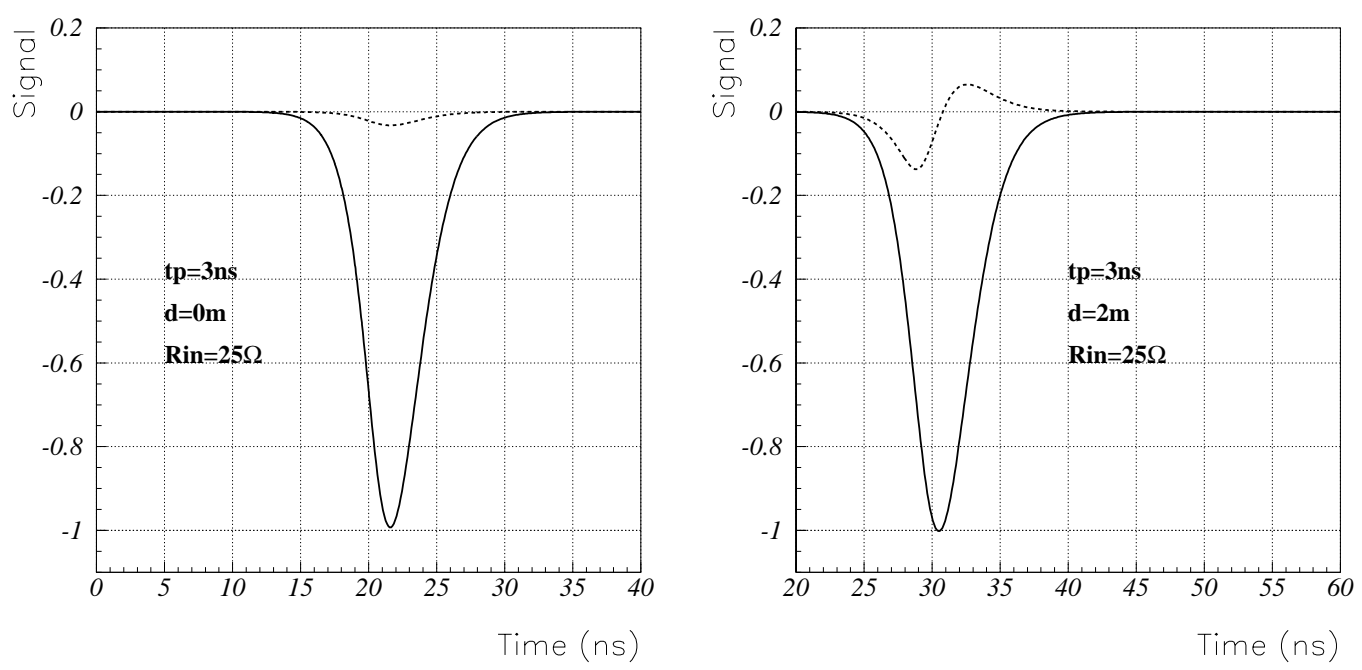

Figure 13: Signal and crosstalk to the first neighbour strip for two different distances of the induced current from the amplifier. The solid line shows the signal strip, the dotted line the crosstalk. Close to the amplifier the dispersion is small and the crosstalk signal has the same shape as the original one (first figure). For larger distances the shape changes and the crosstalk increases (second figure). The integral over the crosstalk signal does not change as a function of the distance (Eq. 10) and therefore the signal will become more bipolar for larger distances. 


\section{$7 \quad$ Frequency Dependence and Losses}

For all the previous studies we neglected losses and assumed that $\hat{\mathbf{L}}$ and $\hat{\mathbf{C}}$ are independent of frequency. A frequency dependence of these two matrices will introduce dispersion in addition to the modal dispersion effect discussed earlier. For conductors with small losses $\hat{\mathbf{L}}$ will be frequency independent. The matrix $\hat{\mathbf{C}}$ however will be affeced by a frequency dependence of the permittivity $\epsilon$ of the surrounding medium. Most dielectrics are reported to show no frequency and loss effects up to the GHz range and since amplifiers used for RPCs rarely exceed a bandwidth of $200 \mathrm{MHz}$ we should not have to worry about these effects. Bakelite however is a very bad material in that repect and shows losses and frequency effects already far below $1 \mathrm{GHz}$, so we have to check our assumptions carefully.

Two kinds of losses can occur in the given transmission lines. Losses in the conductors that will be represented by the matrix $\hat{\mathbf{R}}$ in Eq. 1 and losses in the surrounding medium (e.g. the Bakelite) which are represented by the matrix $\hat{\mathbf{G}}$ in Eq. 2. In general these losses will cause frequency dependent dispersion and exponential attenuation. A general formalism for lossy multi-conductor transmission lines exists, in this report we will however only discuss the losses for a homogeneous single conductor transmission line (Fig. 14) to estimate the effects. The losses introduce a frequency dependence, so we have to work in the frequency domain. Putting a sine-wave with amplitude $A_{0}$ on the conductor at $z=0$ we find an attenuated and phase shifted sine-wave at position $z$ according to

$$
A(z, t)=A_{0} e^{-(\alpha+i \beta) z} e^{\omega t} \quad \alpha+i \beta=\sqrt{(R+i \omega L)(G+i \omega C)}
$$

where $\alpha$ is the attenuation factor that we are interested in. For small losses i.e. $R<<\omega L$ and $G<<\omega C$ the factor can be approximated by

$$
\alpha \approx \frac{1}{2} \frac{R}{Z_{C}}+\frac{1}{2} G Z_{C} \quad Z_{C}=\sqrt{\frac{L}{C}}
$$

where $Z_{C}$ is the characteristic impedance in the limit of high frequencies. The attenuation length $l_{\text {att }}$ is then given by $1 / \alpha$.

\subsection{Losses due to $R$}

The losses due to the resistance of the readout electrodes are given by the DC resistance at low frequencies and by the skin effect at high frequencies. Assuming that all the current is flowing within one skin-depth of the conductor [4] we find the resistance numbers given in Fig. 15 for the RPC geometry in Fig. 9. At a frequency of $1 \mathrm{GHz}$ the resistance of signal and guard strip (copper) is about 0.2 and $10 \Omega / \mathrm{m}$. The characteristic impedance of the RPC strips in Fig. 9 is about $20 \Omega$ and $120 \Omega$ so we find attenuation lengths of $200 \mathrm{~m}$ and $24 \mathrm{~m}$ for signal and guard strip wich is certainly negligible for RPCs of a few metres length.

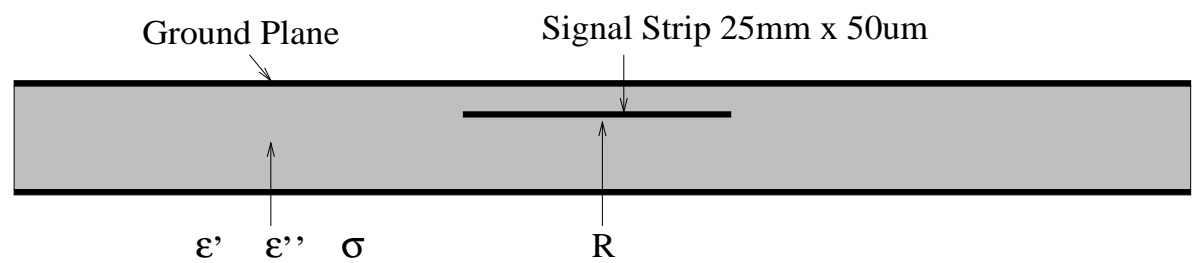

Figure 14: Homogeneous transmission line with a single lossy conductor and a lossy surrounding medium. 


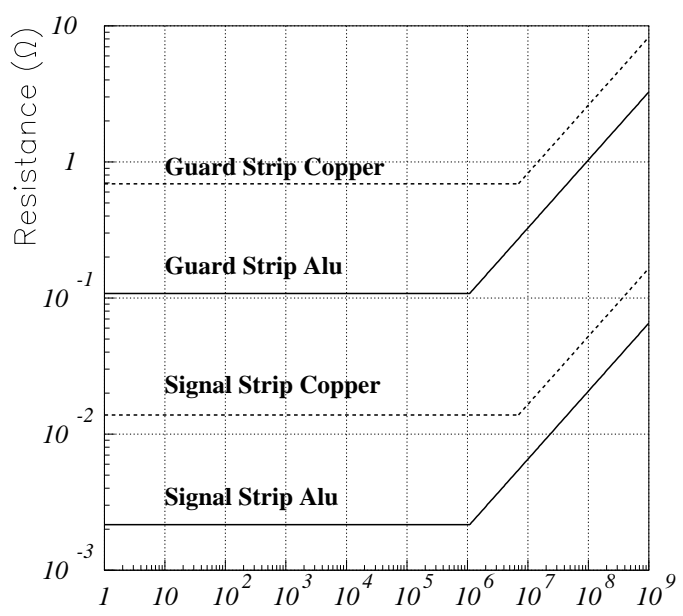

Figure 15: Conductor resistance assuming all the current to flow within one skin-depth for the RPC geometry in Fig. 9.

\subsection{Losses due to $G$}

The losses to the surrounding medium are due to conduction losses and polarization losses. Conduction losses due to free charge in the dielectric medium are characterized by $\sigma$, polarization losses due to bound charge in the dielectric are characterized by an imaginary permittivity $\epsilon_{i}$. They can be included in the calculation by introducing a complex permittivity

$$
\epsilon=\epsilon_{r}-i\left[\epsilon_{i}+\frac{\sigma}{\omega}\right]
$$

Calculating the complex capacitance (or capacitance matrix) $\hat{\mathbf{C}}_{I}$ for this complex permittivity, the capacitance matrix $\hat{\mathbf{C}}$ and conductance matrix $\hat{\mathbf{G}}$ are given by

$$
\hat{\mathbf{C}}=\operatorname{Re}\left[\hat{\mathbf{C}}_{I}\right] \quad \hat{\mathbf{G}}=-\omega \operatorname{Im}\left[\hat{\mathbf{C}}_{I}\right]
$$

so for a homogeneous single conductor transmission line like in Fig. 14 we have

$$
G=G_{\text {cond }}+G_{\text {pol }}=\frac{\sigma}{\epsilon_{r}} C+\omega \frac{\epsilon_{i}}{\epsilon_{r}} C=\frac{\sigma}{\epsilon_{r}} \frac{1}{v Z_{C}}+\omega \frac{\epsilon_{i}}{\epsilon_{r}} C
$$

The effect from $G_{\text {cond }}$ can best be estimated by inserting it into Eq. $13(R=0)$ which gives

$$
\alpha \approx \frac{\sigma}{2 \epsilon_{r} v}
$$

The Bakelite used for RPCs usually has a conductivity of $\sigma<10^{-8} \mathrm{~S} / \mathrm{m}$ so for a line with a permittivity of $\epsilon_{r}=\epsilon_{0}$ and $v=c$ we find an attenuation length $l_{\text {att }}>5 \times 10^{5} \mathrm{~m}$. Therefore the effect from the conductivity $\sigma$ can be completely neglected.

The effect from polarization loss $G_{p o l}$ can best be estimated by rewriting the expression in Eq. 12 as

$$
\alpha+i \beta=\sqrt{(R+i \omega L)\left(G_{p o l}+i \omega C\right)}=\sqrt{(R+i \omega L)\left(\frac{\epsilon_{i}}{\epsilon_{r}}+i\right) \omega C}
$$


The ratio $\epsilon_{i} / \epsilon_{r}$ is often referred to as dissipation factor or loss tangent tan $\delta_{\epsilon}$. As long as the loss tangent is much smaller than unity the polarization losses can be neglected. The loss angle of Bakelite varies significantly with frequency and is also different for different kinds of Bakelite. In general the loss tangent is $<0.001$ below $1 \mathrm{GHz}$ for most dielectric materials, but as discussed before one has to be careful with Bakelite.

For all our previous studies we only assumed $\epsilon_{r}=10$ and $\epsilon_{i}, \sigma=0$. A comparison of this model with measurements on an actual RPC is shown in Fig. 16. A voltage sine wave was connected to one strip and the amplitude on the first and second neighbour was measured. The measurement errors were estimated by checking the sensitivity of the measurement results to external variations (changing the orientation of the RPC, grounding etc.). The agreement is acceptable for frequencies $f<200 \mathrm{MHz}$, which is the frequency range that is important for realistic amplifiers.
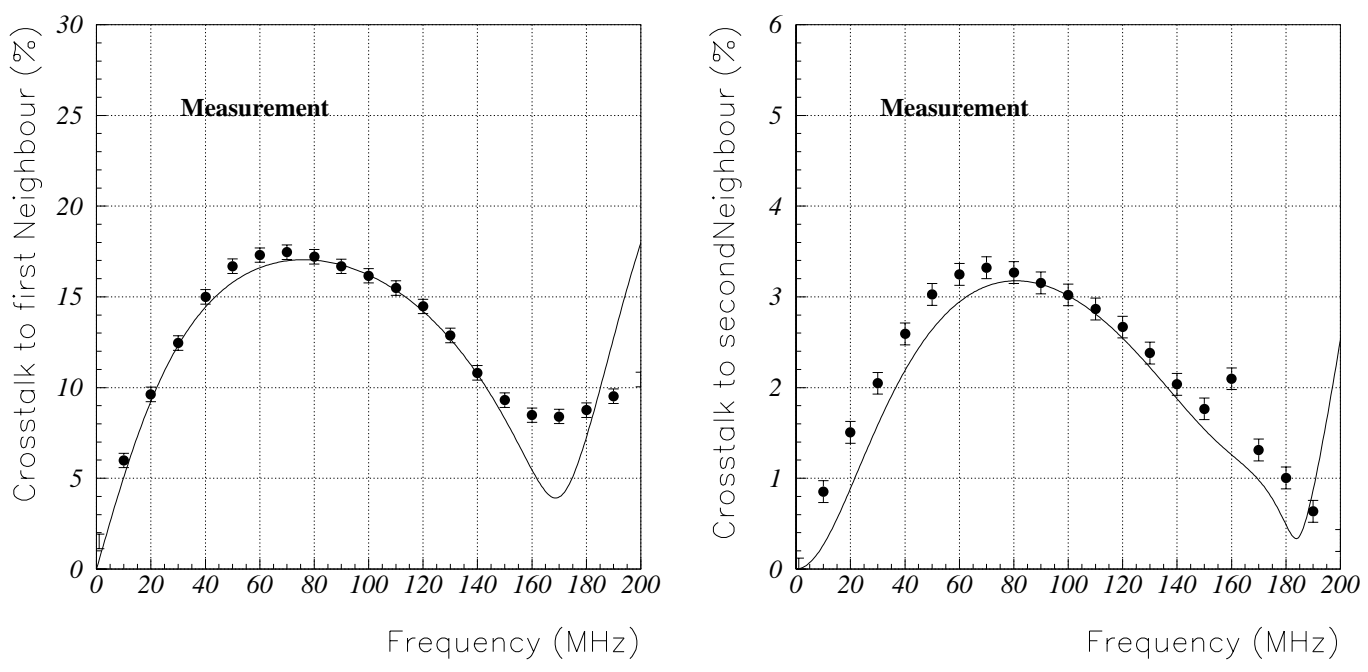

Figure 16: Measurement of the crosstalk for an RPC with the geometry similar to Fig. 9. A voltage sine wave was put on one strip and the amplitude on the first and second neighbour strip was measured. The Bakelite was assumed to have $\epsilon=10$, losses were neglected. The solid line shows the simulation, the points show the measurement. For frequencies $<200 \mathrm{MHz}$ the agreement is acceptable. 
We studied signal propagation in RPCs by analyzing the explicit time domain solution of a lossless $N$-conductor transmission line. Measurements on a RPC prototype show that this model is applicable in the frequency which realistic amplifiers are sensitive to. The RPC is completely defined by the 'per unit length' capacitance and inductance matrix that were calculated with Maxwell [5]. The symbolic solution for an induced current pulse $I^{0}(t)$ at some position $z=z_{0}$ along the strip is then completely defined and the reflected and measured pulses at the line ends can be calculated with a very elegant matrix formalism. For this report this was done with Mathematica [6].

The formalism allows some general conclusions:

- To avoid reflections on one side of the RPC the strips theoretically have to be interconnected by $\frac{1}{2} N(N+1)$ resistors. The realistic number of interconnections has to be decided depending on specifications.

- Since the RPC is an inhomogeneous transmission line the signals propagate as a linear superposition of pulses that are equal to the original induced signal and travel with $N$ different velocities. Therefore we find signal dispersion and dependence of the crosstalk amplitude and shape on amplifier speed, signal shape and position of the induced signal along the strip.

- The crosstalk is lowest if the amplifier input resistance is as low as possible, the strips are not interconnected on the amplifier side and the amplifiers are as slow as possible. It is therefore important to chose the slowest possible electronics that is still compatible with timing requirements.

Specific to the RPC geometry shown in Fig. 9 we can conclude:

- For a strip length of $2 \mathrm{~m}$ the crosstalk to the first neighbour ranges from $3.3 \%$ to $13.7 \%$ for an amplifier peaking of $3 \mathrm{~ns}$ and from $3.3 \%$ to $7.4 \%$ for a peaking time of $10 \mathrm{~ns}$ $\left(R_{\text {in }}=25 \Omega\right)$.

- Since the direct coupling to the second neighbour is very small the crosstalk to the second neighbour happens mainly through the first one and is therefore approximately given by the square of the above numbers. The result is $0.15 \%$ to $1.87 \%$ for 3 ns peaking time and $0.15 \%$ to $0.76 \%$ for $10 \mathrm{~ns}$ peaking time.

- Theoretical considerations show that losses due to conductor resistance, conductivity $\sigma$ and imaginary permittivity $\epsilon_{i}$ of the Bakelite should be small within the bandwidth of applicable amplifiers i.e. $<250 \mathrm{MHz}$.

- Measurements on a prototype confirm that in this frequency range the losses can indeed be neglected. In general however Bakelite is a material that is not very well defined and therefore the losses have to be watched carefully.

We would like to thank Giovanni Carboni for providing an RPC and for many useful discussions. We also thank Christoph Posch for many important suggestions. 


\section{References}

[1] M. Adinolfi et. al, Proposal for the RPC muon detector of LHCb, LHCb note, LHCb-2000053, CERN 2000.

[2] ATLAS Muon Spectrometer Technical Design Report, CERN-LHCC-97-22, ATLAS TDR 10, CERN 1997.

[3] Werner Riegler, RPC Simulations, LHCb note, LHCb Muon-2000-112, CERN 2000.

[4] Clayton R. Paul, Analysis of Multiconductor Transmission Lines, John Wiley \& Sons 1994.

[5] MAXWELL 2D Extractor, Version 4.0.05, 1984-1999 Ansoft Corporation.

[6] Mathematica 4.0, Wolfram Research 1999. 